\title{
Filmes Tubulares de Compósitos de Termoplásticos Pós-consumo: Análise Térmica e Mecânica
}

\author{
Ruth M. C. Santana \\ Divisão de Química, IPT, USP \\ Sati Manrich \\ Depto. de Engenharia de Materiais, UFSCar
}

\begin{abstract}
Resumo: Compósitos ternários de resíduos pós-consumo de polipropileno (PP), poliestireno de alto impacto (HIPS) e carbonato de cálcio $\left(\mathrm{CaCO}_{3}\right)$ foram preparados para obter filmes de papel sintético e avaliar suas propriedades térmicas e mecânicas. Estes filmes compósitos altamente carregados com 10, 20 e $30 \%$ em peso de $\mathrm{CaCO}_{3}$ foram extrudados na extrusora sopro de filme (matriz tubular), em duas condições de velocidade da rosca: 32 e $51 \mathrm{rpm}$. A orientação dos filmes compósitos foi determinada por meio dos parâmetros das razões de processo: $\mathrm{R}_{1}$ (ao longo da direção da máquina), $\mathrm{R}_{\mathrm{t}}$ (na direção transversal da máquina) e $\mathrm{R}_{\mathrm{c}}$ (razão entre $\mathrm{R}_{\mathrm{L}} / \mathrm{R}_{\mathrm{t}}$ ). Ensaios de caracterização por Calorimetria diferencial de varredura (DSC) e resistência à tração e ao rasgo foram usados para estudar as propriedades térmicas e mecânicas dos filmes, observando-se a dependência da composição e do grau de orientação. Resultados da análise térmica por DSC indicaram que a concentração de carga, assim como a orientação do filme, exerce pouca influência tanto na temperatura de fusão Tm quanto no grau de cristalinidade. Resultados do ensaio de tração mostraram que o módulo de elasticidade dos filmes compósitos com maior concentração de carga diminui na direção longitudinal e aumenta na transversal. Quanto à resistência ao rasgo, o efeito do aumento da concentração de carga e de orientação foi o de incrementar essa propriedade.
\end{abstract}

Palavras Chave: Compósitos, filmes, reciclagem, polipropileno, poliestireno de alto impacto.

\section{Blown Films of Post-Consumer Thermoplastic Composites: Thermal and Mechanical Analyses}

Abstract: Ternary composites from post-consumer waste of polypropylene (PP), high impact polystyrene (HIPS) and calcium carbonate $\left(\mathrm{CaCO}_{3}\right)$ were prepared to obtain films of synthetic paper and to evaluate their thermal and mechanical properties. These composites filled with 10, 20 and $30 \% \mathrm{wt}$ of $\mathrm{CaCO}_{3}$ were film blown extruded under two conditions of screw rotation: 32 and $51 \mathrm{rpm}$. The composite film orientation was determined with the parameters along the longitudinal axis (Rl), transverse axis (Rt) and Rl/Rt ratio (Rc). Differential scanning calorimetry (DSC) and tensile and tear strength tests were used to study the thermal and mechanical properties of the films, showing their dependence on composition and orientation degree. Composite films with higher filler concentration exhibited a decreased tensile strength, but a less significant effect on tensile modulus. The tear strength was strongly dependent on the degree of film orientation.

Keywords: Composites, films, recycling, polypropylene, high impact polystyrene.

\section{Introdução}

As propriedades dos polímeros carregados/reforçados diferem em muitos aspectos dos não carregados e dependem essencialmente das características físico-químicas das cargas, sendo os seguintes fatores os mais importantes: forma geométrica, tamanho médio e distribuição de tamanho, área superficial específica, características de empacotamento, natureza química da superfície e energia superficial, pureza química, higroscopicidade e abrasividade das partículas na interação entre o polímero e a carga ${ }^{[1-3]}$.

Segundo a classificação das cargas, estas podem ser fibrosas ou não, orgânicas ou inorgânicas e naturais ou sintéticas ${ }^{[2]}$. Neste caso específico trataremos da carga inorgânica, onde, dependendo da capacidade de reforço, pode ser classificado como (1) carga reforçante ou (2) inerte ou de enchimento ${ }^{[2-4]}$. Cargas inorgânicas inertes adicionadas ao polímero alteram as propriedades deste, aumentando a rigidez, dureza, densidade, temperatura de deflexão térmica (HDT) e estabilidade dimensional, menor retração de moldagem, menor dependência das propriedades com a temperatura, menor resistência à tração e fluência e menor custo $^{[2,5]}$. Por outro lado, as cargas reforçantes ou ativas apresentam um custo superior comparado ao das cargas inertes ou de enchimento, que melhoram suas propriedades mecânicas, tal como a resistência à tração. A resistência ao impacto é variada, pois em geral a presença de cargas rígidas em matrizes dúcteis (como polipropileno e ABS) reduz

Autor para correspondência: Sati Manrich, Departamento de Engenharia de Materiais, UFSCar, Caixa Postal 676, CEP: 13565-905, São Carlos, SP. Email:sati@power.ufscar.br 
a tenacidade do material, enquanto que cargas em matrizes frágeis (como poliestireno) podem resultar em resistência ao impacto superior ${ }^{[2,6,7]}$.

O polipropileno (PP) isotático carregado com carga inorgânica tais como carbonato de cálcio, talco, mica, caulim, sílica, amianto entre outros ${ }^{[1,2,5,6,8]}$, tem sido amplamente usado em muitas aplicações industriais devido a sua variedade de propriedades físicas, boas propriedades mecânicas e baixo custo. Algumas das cargas inorgânicas nos compósitos de PP freqüentemente afetam a cristalização da fase matriz, aumentando a temperatura de cristalização (Tc) e/ou o seu grau de cristalinidade, portanto, atuando como agente nucleante ${ }^{[2,4,5,9]}$.

Compósitos ternários a partir de uma blenda e cargas particuladas são materiais atrativos para numerosas aplicações de engenharia e suas propriedades mecânicas são determinadas não só pela sua composição, mas também pela morfologia da fase presente, em particular da dispersão relativa dos componentes ${ }^{[6]}$. Uma das aplicações destes compósitos altamente carregados é o papel sintético. Publicações sobre papel sintético a partir de plástico pós-consumo, ainda são muito escassas em revistas científicas e/ou tecnológicas nacionais e internacionais. Por outro lado, a maior fonte de informação obtida de papel sintético a partir de resina virgem ainda é através de patentes, sendo as primeiras patentes pioneiras de Mannar et al., $(1994)^{[10]}$, e Desai, (1994) $)^{[11]}$.

Na maioria das patentes publicadas sobre papel sintético, o polímero mais usado é o polipropileno (PP), em filmes multicamadas ${ }^{[12-15]}$, processadas por coextrusão ${ }^{[16-19]}$ ou extrusão filme-plano com sistema de biorientação ${ }^{[20]}$, entre outros. Em todas as patentes é verificado que a carga inorgânica empregada como outro dos componentes principais do papel sintético é o $\mathrm{CaCO}_{3}$, com concentrações de 10 a $75 \%$, com o objetivo principal de promover opacidade, rigidez e maior rugosidade, características principais do papel celulósico. A presença da carga também promove a formação de microcavitações quando o filme-compósito é submetido ao processo de orientação e como conseqüência um aumento na opacidade, uma vez que, com a maior concentração de vazios, a luz incidente é difratada em maiores proporções ${ }^{[10,12,21-23]}$. Segundo Ohno et al., (1995), filmes compósitos planos (não tubulares) biorientados, com concentração de $\mathrm{CaCO}_{3}$ de 10 a $35 \%$ em peso (tamanho médio de partícula de 0,05 a $8 \mu \mathrm{m}$ ), apresentam em média uma percentagem de vazio gerado na faixa de 5 a $55 \%{ }^{[12]}$.

Por outro lado, o processamento de filme tubular (usado neste trabalho) apresenta características singulares se comparado à maioria das linhas de extrusão, devido à formação de uma bolha de filme. A geometria da bolha é controlada pelas diversas velocidades da máquina e pelo volume interno de ar, de forma a produzir filmes. Quando a extrusora se encontra operando em equilíbrio, há quatro parâmetros para controle de processo que determinam a geometria da bolha ${ }^{[24]}$, elas são: (1) velocidades dos rolos de arraste (Vp), (2) velocidade da rosca (Vr), (3) volume de ar de resfriamento (Var) e (4) pressão de ar de sopro (P). As combinações destas variáveis defi- nem a geometria particular da bolha, tais como: (1) altura da linha da névoa, (2) espessura do filme e (3) diâmetro da bolha.

Segundo Cantor ${ }^{[24]}$ os parâmetros de processamento exercem forte influência sobre as propriedades do filme, tendo sido as razões de processo consideradas neste estudo. A razão de extração, definida como a velocidade dos rolos de arraste dividida pela velocidade de fluxo da resina fundida, fornece uma indicação sobre o grau de estiramento na direção da máquina e, portanto, a orientação do filme nessa direção. A taxa de sopro, definida como sendo o diâmetro da bolha dividido pelo diâmetro da matriz, fornece uma indicação sobre o grau de estiramento na direção transversal, ou seja, orientação. A taxa de conformação, que é a razão de extração dividida pela razão de sopro, representa uma medida do grau de proporção de orientação entre as direções da máquina e transversal.

Por outro lado, entre as variáveis moleculares que interferem no comportamento reológico do polímero durante o processamento, além de interferir nas propriedades finais dos filmes estão: a massa molar, a distribuição de massa molar, o tipo e a quantidade de ramificações e distribuição destas ramificações ${ }^{[25]}$. Segundo Rosato\&Rosato, $(1988)^{[25]}$, uma alta massa molar induz a uma maior orientação que influencia em melhores propriedades finais dos filmes, como resistência à tensão, resistência à fluência, resistência ao impacto, resistência térmica e resistência por degradação quando em contato com solventes.

Os processos de orientação são muito empregados na modificação de algumas propriedades importantes de filmes, como propriedades mecânicas, óticas e de barreira a gases, além de alterarem a espessura e a flexibilidade dos mesmos ${ }^{[26]}$. No geral, qualquer polímero termoplástico pode ser orientado e este processo de orientação ocorre geralmente um pouco acima da temperatura de transição vítrea $(\mathrm{Tg})$.

Existem três componentes reológicos distintos que atuam sobre o polímero no processo de orientação: E1, deformação elástica instantânea causada pela deformação das ligações químicas primárias. Essa deformação é completamente recuperada quando a tensão é retirada; E2, deformação molecular ou alinhamento das moléculas e E3, fluxo viscoso irrecuperável, causado pelas moléculas que escorregam umas sobre as outras.

$\mathrm{O}$ valor relativo de cada componente depende principalmente da temperatura, tempo, e do nível de tensão aplicada no processo ${ }^{[26]}$. Portanto quanto maior a tensão de estiramento aplicada, maior será o valor da componente reológica E2, sendo que no tempo inicial os valores de E2 é alto e de E3 baixo. Se o material for resfriado rapidamente, a estrutura molecular alinhada será congelada e o polímero estará orientado. Após todo o desdobramento das moléculas, o componente E3 pode atuar, sendo controlada não só pela temperatura e nível de tensão aplicada, mas também pelo tempo. Porém a temperatura influencia na recuperação de E2, portanto os dois processos são competitivos (estiramento e recuperação) ${ }^{[27]}$.

Considerando todas essas variáveis de processo, foi realizado um estudo sobre filmes de compósitos PP/HIPS/ $\mathrm{CaCO}_{3}$ pós-consumo para aplicação em papel sintético, obtidos por 
extrusão sopro de filme tubular, em que o presente trabalho visa apresentar os resultados referentes às propriedades térmicas e mecânicas desses filmes.

\section{Experimental}

Os materiais plásticos pós-consumo usados foram o polipropileno (PP) e o poliestireno de alto impacto (HIPS) provenientes de garrafas de água mineral e de copos descartáveis respectivamente, da coleta seletiva do Programa de Entrega Voluntária (PEV) da UFSCar. Como carga foi usado o carbonato de cálcio $\left(\mathrm{CaCO}_{3}\right)$, de tamanho médio de partícula de $8 \mu \mathrm{m}$, (Inacarb-700) fornecido pela Quimbarra-Brasil. O PP e o HIPS foram pré-moídos, lavados, secos e moídos novamente. Três compósitos ternários de PP/HIPS $/ \mathrm{CaCO}_{3}$ com proporção mássica de 60/30/10, 60/20/20 e 60/10/30 na forma de pellets foram preparados na extrusora dupla rosca Werner Pfleiderer (modelo ZSK 30). Filmes tubulares dos compósitos foram processados na extrusora mono-rosca CIOLA IF40, com perfil de temperatura de 190, 200, 210, 210 e $220^{\circ} \mathrm{C}$ e velocidade da rosca de 32 e $51 \mathrm{rpm}$.

O grau de orientação dos filmes compósitos foi determinado segundo a metodologia de Cantor ${ }^{[24]}$. Neste caso, R1 definida como Vp/Vrosca, fornece uma indicação sobre o grau de estiramento na direção da máquina; Rt definida como $\mathrm{Db} /$ Dmatriz, fornece uma indicação sobre o grau de estiramento na direção transversal, e Rc, definida como Rl/Rt, representa uma medida do grau de proporção de orientação entre as direções longitudinal (da máquina) e transversal.

A caracterização térmica foi realizada através do teste de calorimetria diferencial de varredura - DSC (Perkin Elmer, modelo Pyres-1), na faixa de temperatura de 30 a $200^{\circ} \mathrm{C}$ e com taxa de aquecimento de $10^{\circ} \mathrm{C} / \mathrm{min}$, onde o grau de cristalinidade foi determinado considerando a composição do $\mathrm{PP}(\% \mathrm{PP})$ na mistura, segundo a equação $\left(\mathrm{Xc}=\Delta \mathrm{Hf}_{\text {amostra }} \% \mathrm{PP} /\right.$ $\left.\Delta \mathrm{Hf}_{(100 \% \text { cristalino })}\right)$. A caracterização mecânica por meio do ensaio de tração dos filmes foi realizada na máquina Universal de Ensaios Instron, modelo 122, segundo a norma ASTM D882-90.

A determinação de resistência ao rasgo foi realizada segundo a norma ASTM D689-92, onde descreve os procedimentos utilizados para a medida da força média em gramas, necessária para o rasgamento simultâneo de uma ou mais folhas de papel ou filmes, por uma determinada distância, após o rasgo ter sido iniciado. Para a realização do teste foi utilizado o aparelho de rasgamento tipo Elmendorf que mede o trabalho realizado no rasgamento das folhas, calculado por meio da perda da energia potencial de um pêndulo. Para complementar este estudo, foi realizada a caracterização morfológica da superfície dos filmes utilizando microscópio eletrônico de varredura, marca Lo, modelo Stereoscan 440.

\section{Resultados e Discussão}

Neste trabalho, os parâmetros velocidade de puxamento (Vp), pressão de ar de sopro (Par), volume de ar de resfriamento (Var), assim como o perfil de temperatura. foram mantidos constantes. A determinação do diâmetro da bolha $(\mathrm{Db})$ foi através da medição da largura do filme achatado LF (layflat width) após este ter passado através dos rolos de arraste, isto é $\mathrm{Db}=(2 \mathrm{LF}) / \pi$. Na Tabela 1 são mostradas as razões de processamento dos filmes tubulares: razão de extração $(\mathrm{Rl})$, razão de sopro $(\mathrm{Rt})$ e razão de conformação (Rc).

Os filmes compósitos apresentaram maior grau de orientação na direção longitudinal do que na direção transversal $(\mathrm{Rl}>\mathrm{Rt})$, sendo que a velocidade de puxamento de polias $(12,5 \mathrm{~m} / \mathrm{min})$ é o parâmetro que confere esta propriedade. Os filmes extrudados a $51 \mathrm{rpm}$ apresentaram menor valor de Rl que os processados a $32 \mathrm{rpm}$, nas três composições, devido à maior vazão mássica, o que dificultaria a orientação do filme. Já no caso dos valores de Rt, estas não apresentaram muita variação entre as duas condições de velocidades da rosca, já que a orientação periférica (transversal) é mais influenciada pelo ajuste da pressão de ar de sopro, sendo que neste caso, foi mantido constante. Estes resultados podem ser melhor visualizados nos valores de Rc a $51 \mathrm{rpm}<32$ rpm, que é diretamente proporcional à variação da orientação preferencial longitudinal.

Em relação à concentração de carga no filme compósito, um aumento desta apresenta maior influência na orientação transversal, onde se observa uma diminuição da Rt, devido ao aumento da concentração da carga que influenciou na dificuldade do seu fluxo extensional e, portanto, resultando no decréscimo de sua orientação para ambas as condições, sendo mais visível nos processados a $32 \mathrm{rpm}$. Por outro lado, o valor de Rc aumenta com a concentração da carga para ambos os casos; onde se observa um acréscimo maior da orientação dos filmes processados a $51 \mathrm{rpm}(\mathrm{F} 2$ a F3), de $31 \%$, do que os processados a $32 \mathrm{rpm}$, de $13 \%$.

Tabela 1. Grau de orientação na direção longitudinal (Rl), transversal (Rt) e a razão de orientação entre ambas as direções Rc=R1/Rt dos filmes compósitos.

\begin{tabular}{ccccccc}
\hline $\begin{array}{c}\text { Filme compósito } \\
\text { (PP/HIPS/carga) }\end{array}$ & $\begin{array}{c}\mathbf{V}_{\text {rosca }} \\
(\mathbf{r p m})\end{array}$ & $\begin{array}{c}\text { Espessura } \\
(\boldsymbol{\mu m})\end{array}$ & $\begin{array}{c}\mathbf{D}_{\text {bolha }} \\
(\mathbf{c m})\end{array}$ & $\mathbf{R l}$ & $\mathbf{R t}$ & $\mathbf{R c}$ \\
\hline \multirow{2}{*}{ F1 (60/30/10) } & 37 & $61 \pm 1$ & 12,4 & 2,26 & 1,24 & 1,82 \\
& 51 & $66 \pm 5$ & 12,1 & 1,66 & 1,21 & 1,37 \\
\hline \multirow{2}{*}{ F2 (60/20/20) } & 32 & $59 \pm 1$ & 10,5 & 2,65 & 1,06 & 2,50 \\
& 51 & $70 \pm 2$ & 12,1 & 1,66 & 1,21 & 1,37 \\
\hline \multirow{2}{*}{ F3 (60/10/30) } & 32 & $61 \pm 1$ & 9,4 & 2,65 & 0,94 & 2,82 \\
& 51 & $92 \pm 1$ & 9,2 & 1,66 & 0,92 & 1,80 \\
\hline
\end{tabular}


Em relação à análise térmica por DSC dos filmes compósitos processados a 32 e $51 \mathrm{rpm}$ observa-se que os filmes processados a $51 \mathrm{rpm}$ apresentaram uma temperatura de fusão $(\mathrm{Tm})$ ligeiramente superior aos processados a 32 rpm, cuja diferença pode ser considerada desprezível, por ser menor do que $1^{\circ} \mathrm{C}$. Com relação à concentração do $\mathrm{CaCO}_{3}$ no compósito, observa-se também um ligeiro acréscimo de Tm $\left(\sim 1^{\circ} \mathrm{C}\right)$, que também pode ser considerado desprezível. Estes resultados indicam que a concentração da carga, assim como a orientação do filme exerce pouca influência na temperatura de fusão do compósito, como pode ser visualizado na Tabela 2.

Poderia-se esperar que a alta concentração de carga $\mathrm{CaCO}_{3}$ e a presença da fase amorfa tanto do HIPS como do próprio PP, resultaria em diminuição significativa do grau de cristalinidade (Xc) dos filmes de compósitos, o que não ocorreu. Em relação ao efeito de $\mathrm{CaCO}_{3}$, observa-se um pequeno decréscimo do grau de cristalinidade com o aumento de sua concentração em todos os filmes. Isto foi atribuído ao fato de que o movimento das macromoléculas para a ordenação, formando fase cristalina, tenha sido dificultado cada vez mais à medida que se aumentava a concentração de carga, como pode ser visualizado na Figura 1.

Observa-se que o Xc dos filmes processados a $32 \mathrm{rpm}$ é ligeiramente superior ao dos processados a $51 \mathrm{rpm}$ como mostrado na figura 1. Este fato poderia ser explicado por duas possíveis hipóteses: a primeira, é que o menor fluxo mássico (massa de resina fundida), possibilita um maior "desamaranhamento" das moléculas poliméricas do que com maior fluxo mássico (51 rpm), mantendo a velocidade de arraste constante $(\mathrm{Vp})$. A segunda, é que o maior fluxo mássico, indica um menor tempo de residência da massa fundida na rosca, influenciando na má dispersão e distribuição das partículas da carga., o que teria acarretado numa velocidade de arraste $(\mathrm{Vp})$, mantida constante, abaixo da necessária para manter a orientação. Este resultado pode ser confirmado pelos resultados da Tabela 1, em que os filmes compósitos processados a $32 \mathrm{rpm}$ possuem maior grau de orientação em ambas as direções, sendo mais pronunciado na direção da máquina.

Em um estudo realizado por Legros et al.(1998) ${ }^{[28]}$, verificaram que a orientação (Rl) controla a direção do crescimento da fase cristalina. Foi avaliada a influência das razões de processo Rl e Rt, nas propriedades finais dos filmes tubulares e concluíram que há mudanças nas orientações lamelares relativas às mudanças dos parâmetros de processo. Com o aumento de R1, a orientação lamelar na direção transversal se torna mais perfeita; já o aumento de Rt causa desordem no empilhamento das lamelas, concluindo que a velocidade de puxamento não exerce uma orientação potente, mas a orientação (Rl) controla a direção do crescimento da fase cristalina. Como no presente caso, a um fluxo mássico menor com graus maiores de orientação longitudinal o grau de cristalinidade foi também maior, os resultados aqui obtidos estariam corroborando as conclusões apresentadas no estudo de Legros et al.

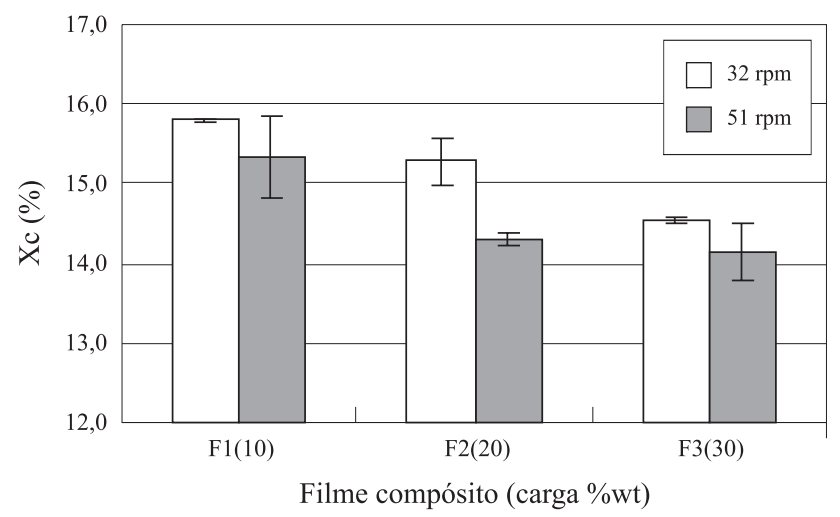

Figura 1. Grau de cristalinidade (\%) dos filmes compósitos tubulares processados a 32 e $51 \mathrm{rpm}$ determinado pela análise de DSC.

Na figura 2 são apresentados os resultados do teste de resistência à tração dos filmes de compósitos nas direções longitudinal e transversal da máquina, onde se observa visivelmente que a resistência máxima à ruptura (Figura 2-a) é maior na direção da máquina do que na direção transversal. Estes resultados são confirmados pelo estudo de Patel et al. ${ }^{[29]}$, onde as tensões de ruptura na direção da máquina foram maiores que os valores na direção transversal, e que as tensões nas direções longitudinal e transversal da máquina são afetadas pela orientação das moléculas da fase amorfa.

Já no caso do módulo de elasticidade, observa-se no geral o mesmo comportamento, isto é, o módulo é maior na direção da máquina do que na transversal, diferentes comportamentos que podem ser relacionados à cristalinidade, organização e tamanho da estrutura cristalina ${ }^{[30]}$. Segundo Legros et $\mathrm{a}^{[28]}$, o módulo sob tração depende da organização da fase cristalina, e quando aumenta a razão de estiramento da máquina (Rl), a orientação lamelar na direção transversal da máquina chega a ser mais perfeita.

Por outro lado, a alta concentração, o tamanho e a aglomeração da carga $\left(\mathrm{CaCO}_{3}\right)$ possivelmente tenham causado uma insuficiente homogeneidade, influenciando fortemente no decréscimo da resistência máxima à ruptura para ambas as condições ( 32 e $51 \mathrm{rpm}$ ), sendo mais pronunciado para os processados a $32 \mathrm{rpm}$. Isto pode ser devido ao fato de que a alta concentração da carga tenha resultado em maiores proporções de aglomerados, que, por atuarem mais efetivamente como pontos concentradores de tensões, a resistência à tração teria sido prejudicada. Além do mais, no processo de estiramento com biorientação em filme tubular, pode originar a formação de microvazios e fissuras a partir das partículas e dos aglomerados de $\mathrm{CaCO}_{3}$ que causariam igualmente a deterioração dessa propriedade.

Quanto ao módulo de elasticidade, que pode ser relacionado à rigidez do material, à exceção do valor obtido para a direção longitudinal nas amostras a $32 \mathrm{rpm}$, é possível verificar pouca influência da proporção de carga adicionada sobre essa propriedade, se considerar a faixa de desvio-padrão, seja em 32 ou $51 \mathrm{rpm}$. Isto poderia indicar que a presença de $10 \%$ de partículas de $\mathrm{CaCO}_{3}$, devido à sua característica rígida e à restrição de movimentos/dobramentos das macromoléculas de PP 

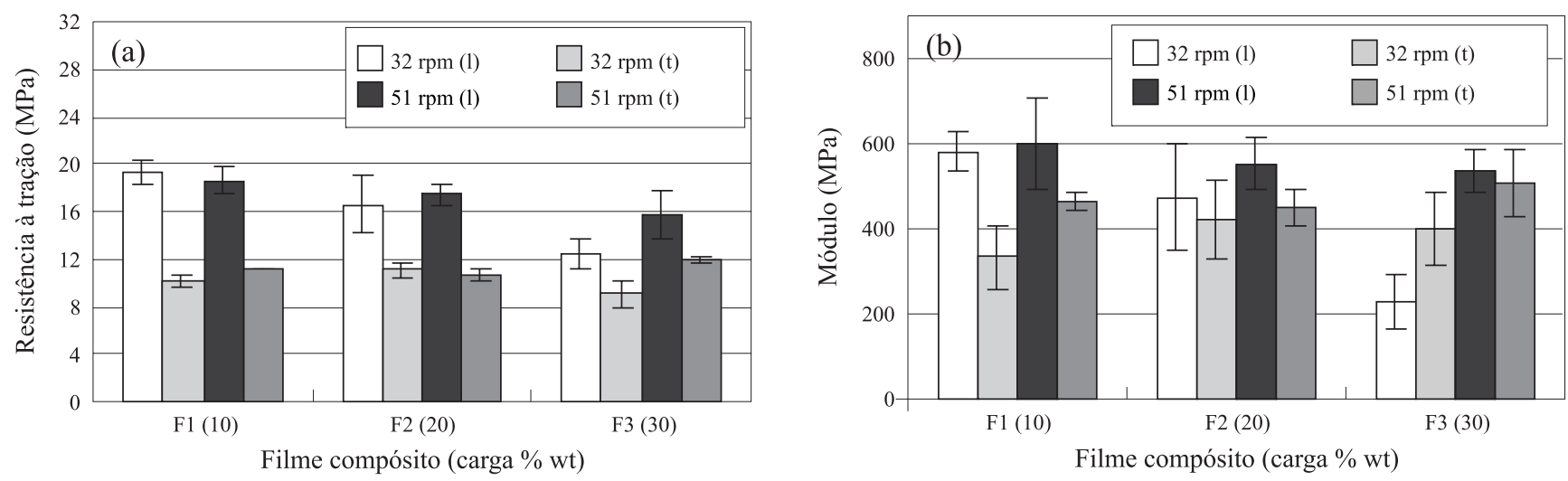

Figura 2. Resultados do teste de tração dos filmes compósitos tubulares processados a 32 e 51 rpm nas direções longitudinal e transversal da máquina (a) resistência máxima à ruptura e (b) módulo de elasticidade.

imposta pelas mesmas, é suficiente para alterar o módulo dos filmes em um nível que posteriores adições dessa carga não acarretam em mudanças significativas nessa propriedade.

Para verificar adequadamente esta influência, uma análise de resultados a partir de um planejamento estatístico de experimentos deveria ser realizada, o que foge ao escopo do presente trabalho. Por outro lado, considerando somente a média dos valores de módulo, observa-se que há uma tendência de aumento na direção transversal e de diminuição na direção longitudinal da máquina, cuja verificação de possíveis razões seria baseada em especulações, uma vez que outras caracterizações não realizadas neste estudo seriam necessárias.
Cabe mencionar que a presença de HIPS no compósito, também pode gerar microvazíos no filme, devido a ser incompatível com $\mathrm{PP}^{[10,12,23]} \mathrm{e}$, portanto, todos estes fatores resultam num decréscimo da resistência à ruptura. Na figura 4 são mostradas as micrografias da superfície dos filmes compósitos. Observam-se algumas aglomerações de carga, especialmente naquelas amostras com maiores proporções (Figura $3 \mathrm{~b}$ e c), onde provavelmente ocorram maiores concentrações de tensões quando os filmes são biorientados. Por outro lado, a presença de HIPS, como polímero incompatível com PP, também influencia na geração de microvazios mostrados nas micrografias Fig. 4 d-e-f, onde se observa a presença de "poros", tanto nas fases poliméricas (blenda
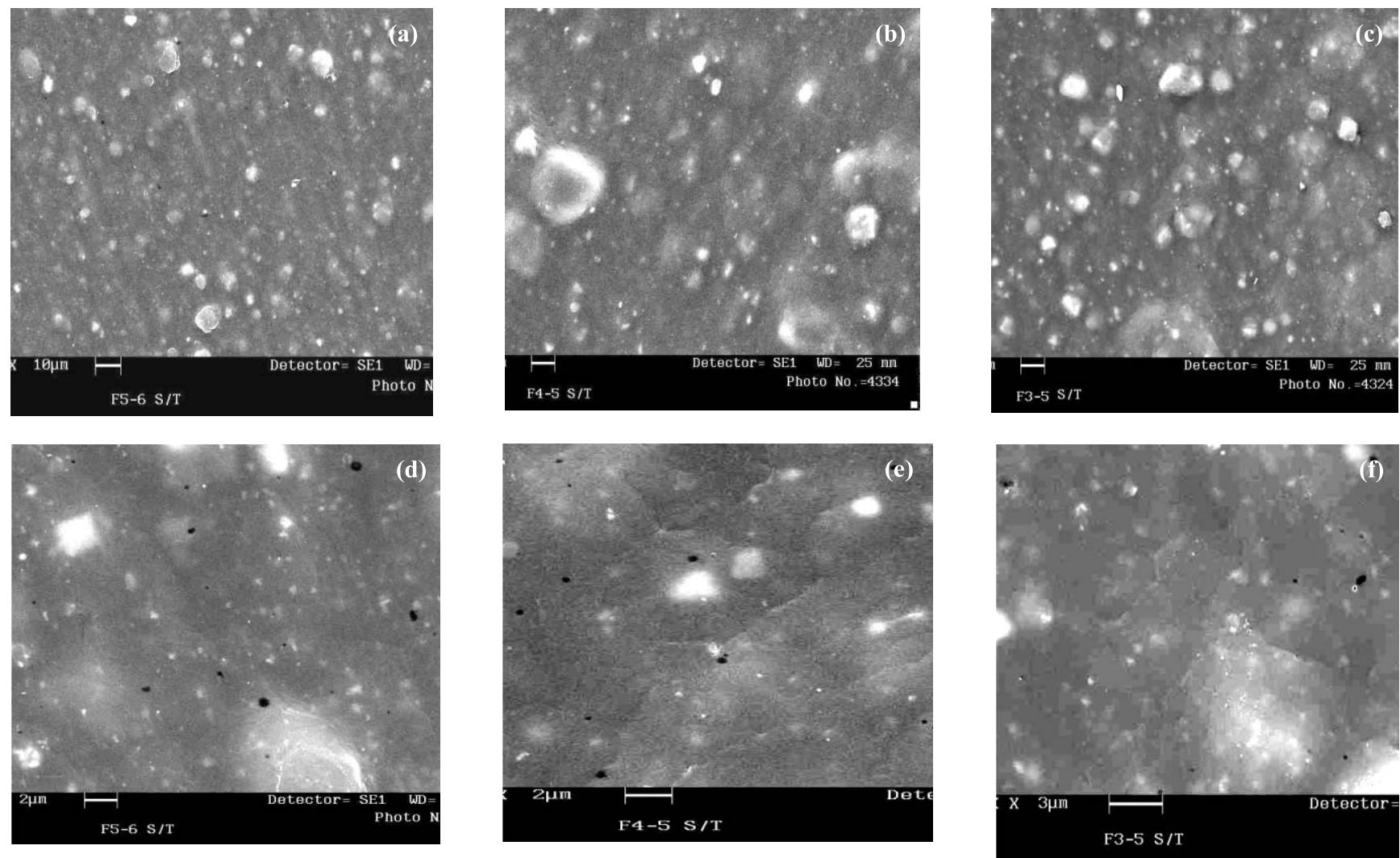

Figura 3 - Micrografias da superfície dos filmes tubulares de compósitos processados a $32 \mathrm{rpm}$ com $10 \%$ (a e d), 20 \% (b e e) e 30 \% (c e f) em massa de $\mathrm{CaCO}_{3}$, com ampliações-dimensão de barras de $1 \mathrm{Kx}-10 \mu \mathrm{m}$ (a-b-c) e $6 \mathrm{Kx}-2 \mu \mathrm{m}$ (d-e-f). 


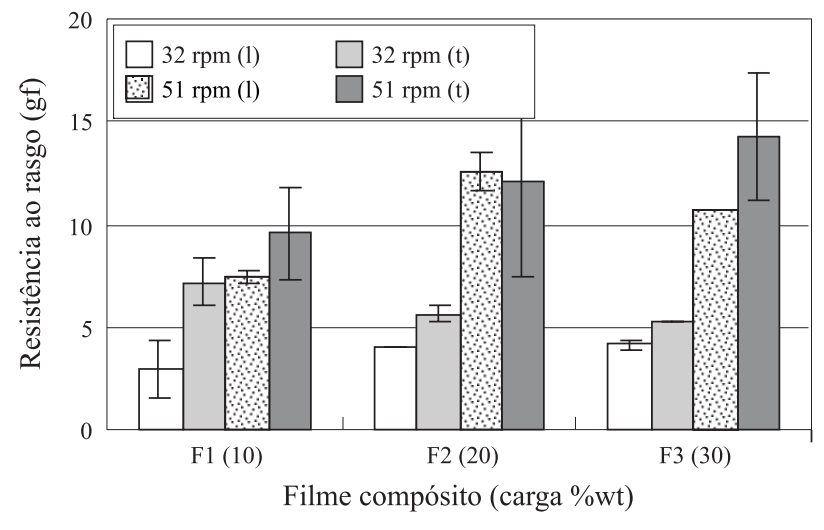

Figura 4. Resultados da resistência ao rasgo dos filmes compósitos tubulares processados a 32 e 51 rpm nas direções longitudinal e transversal da máquina.

polimérica PP/HIPS) quanto na interface fase polimérica / fase dispersa inorgânica (carga).

Na figura 4 são apresentados os resultados do teste de resistência ao rasgo (RR) dos filmes de compósitos nas direções longitudinal e transversal da máquina. Os filmes processados a $51 \mathrm{rpm}$ apresentaram uma RR superior àqueles processados a $32 \mathrm{rpm}\left(\mathrm{RR}_{51 \mathrm{rpm}}>\mathrm{RR}_{32 \mathrm{rpm}}\right)$. Isto é devido à taxa de razão de estiramento: $\mathrm{Rc}$ a $51 \mathrm{rpm}$ ser menor que a 32 rpm, sendo refletida na sua diferença de espessura ( 70-90 $\mu \mathrm{m})$ e $(\sim 60 \mu \mathrm{m})$ respectivamente, que confirma o estudo feito por Krishnaswamy \& Sukhadia ${ }^{[30]}$, relacionado à influência das condições de processamento de estiramento do filme tubular sobre a resistência ao rasgo. Nesse estudo, determinou-se que a RR de filmes soprados apresentam uma forte sensibilidade à taxa de estiramento do filme na direção da máquina, portanto filmes processados com baixo Rl apresentam maior resistência ao rasgo.

Filmes com baixo grau de estiramento apresentam alta RR ao longo da direção da máquina. A força extensional aplicada nas moléculas poliméricas, ao saírem da matriz, tenderá a orientar estas ao longo dessas tensões. A relaxação da conformação estendida das cadeias competirá simultaneamente com sua orientação antes do processo de cristalização. Conseqüente- mente, a altas taxas de extensão na direção da máquina, um maior número de moléculas será orientado ao longo dessa direção antes de sua cristalização. Portanto, é razoável assumir que a fase não cristalina do filme final, em média, tenderia ser mais orientada uniaxialmente, relativo ao plano equibiaxial do filme. Isto resultaria em menores pontos de interações por ligações secundárias e por emaranhamentos entre as moléculas orientadas, o que portanto resultaria numa menor RR ao longo da direção da máquina do filme soprado ${ }^{[28]}$.

Por outro lado, os filmes orientados apresentaram RR maior na direção transversal da máquina do que na longitudinal (RRt $>$ RRl), o que indica que a RRt também é influenciada fortemente pelas condições empregadas, sendo que esta aumenta sistematicamente com o aumento de Rc. Especificamente, maiores taxas de extensão (pequena reorganização estrutural) favorecem a RR na direção transversal ${ }^{[30]}$. Quanto à influência da concentração de $\mathrm{CaCO}_{3}$, observa-se que não há uma tendência definida, uma vez que houve, nos valores de RRl, pequeno aumento ( $32 \mathrm{rpm}$ ) e um máximo para $20 \%$ de $\mathrm{CaCO}_{3}(51 \mathrm{rpm})$, e, nos valores de RRt, diminuição (32 rpm) e aumento (51 rpm) em função da concentração de carga. Isto provavelmente seja decorrente do fato de que tanto o grau de orientação quanto a espessura influam fortemente nessa propriedade, de forma que a influência isolada da adição de carga não fique clara.

Na Tabela 2 são mostrados os valores médios das propriedades térmicas e mecânicas dos filmes tubulares de compósitos processados a 32 e $51 \mathrm{rpm}$ onde se observa que a presença e o aumento da concentração de carga, da espessura e do grau de orientação do filme influenciam de forma geral as propriedades térmicas e mecânicas.

\section{Conclusões}

Segundo os resultados obtidos das razões de processo, os filmes tubulares apresentaram maior orientação na direção da máquina, sendo que Rl é influenciado pela variação da velocidade da rosca (vazão de alimentação). Já no caso do Rt, este é influenciado pela concentração de $\mathrm{CaCO}_{3}$ nos filmes.

A composição e as condições de processamento dos filmes influenciam, em maior ou menor grau, em praticamente todas

Tabela 2. Propriedades térmicas e mecânicas dos filmes compósitos biorientados: temperatura de fusão (Tm), grau de cristalinidade (Xc), módulo (E), resistência máxima a ruptura $(\sigma)$, e resistência ao rasgo $(R R)$ nas direções longitudinal $(L)$ e transversal $(T)$ da máquina.

\begin{tabular}{ccccccccccc}
\hline $\begin{array}{c}\text { Filme } \\
\text { Compósito }\end{array}$ & $\mathbf{R C}=\mathbf{R} / \mathbf{R t}$ & $\begin{array}{c}\mathbf{e} \\
(\boldsymbol{\mu m})\end{array}$ & $\begin{array}{c}\mathbf{T m} \\
\left({ }^{\circ} \mathbf{C}\right)\end{array}$ & $\begin{array}{c}\mathbf{X C} \\
(\boldsymbol{\%})\end{array}$ & $\begin{array}{c}\mathbf{E}_{\mathbf{L}} \\
(\mathbf{M P a})\end{array}$ & $\begin{array}{c}\mathbf{E}_{\mathrm{T}} \\
(\mathbf{M P a})\end{array}$ & $\begin{array}{c}\boldsymbol{\sigma}_{\mathrm{L}} \\
(\mathbf{M P a})\end{array}$ & $\begin{array}{c}\boldsymbol{\sigma}_{\mathrm{T}} \\
(\mathbf{M P a})\end{array}$ & $\begin{array}{c}\mathbf{R R}_{\mathrm{L}} \\
(\mathbf{g F})\end{array}$ & $\begin{array}{c}\mathbf{R R}_{\mathrm{T}} \\
(\mathbf{g F})\end{array}$ \\
\hline F1 (37 rpm) & 1,82 & 60,6 & 161,3 & 15,8 & 582 & 334 & 19,3 & 10,1 & 3,0 & 7,2 \\
F1 (51 rpm) & 1,37 & 66,0 & 161,6 & 15,3 & 600 & 462 & 18,6 & 11,2 & 7,4 & 9,6 \\
F2 (32 rpm) & 2,50 & 58,6 & 161,6 & 15,3 & 474 & 419 & 16,6 & 11,1 & 4,0 & 5,6 \\
F2 (51 rpm) & 1,37 & 70,5 & 162,1 & 14,3 & 553 & 452 & 17,4 & 10,6 & 12,6 & 12,1 \\
F3 (32 rpm) & 2,82 & 60,6 & 162,2 & 14,4 & 228 & 400 & 12,5 & 9,1 & 4,1 & 5,3 \\
F3 (51 rpm) & 1,80 & 92,4 & 162,1 & 14,1 & 537 & 508 & 15,8 & 11,9 & 10,7 & 14,2 \\
\hline
\end{tabular}


as propriedades térmicas e mecânicas avaliadas. Resultados da análise térmica por DSC indicaram que a concentração de carga, assim como a orientação do filme, exerce pouca influência tanto na temperatura de fusão Tm quanto no grau de cristalinidade Xc dos filmes de compósito, entretanto, com uma tendência de aumento de Tm e diminuição de Xc.

A maior concentração de carga influenciou na diminuição da resistência máxima à ruptura, o que foi atribuído a possíveis aglomerados de carga e conseqüentes microvazios, pontos considerados concentradores de tensões. A variação da proporção de carga, de 10 a $30 \%$, resultou em pequena influência sobre o módulo de elasticidade em tração, com tendência ao decréscimo nos valores para a direção longitudinal e acréscimo para a transversal, nas duas condições de rotação utilizadas, 32 e $51 \mathrm{rpm}$. Considerando uma das características mais importantes buscadas nos estudos realizados desses filmes, a de aumento de rigidez relacionada ao módulo de elasticidade, pode-se eleger o compósito com $10 \%$ de $\mathrm{CaCO}_{3}$ como o de maior potencial para aplicação em papel sintético, pelo fato de o filme do mesmo ter apresentado os maiores valores de módulo, independentemente da velocidade de rotação.

Apesar de a concentração de $\mathrm{CaCO}_{3}$ ter indicado efeito significativo sobre a resistência ao rasgo, esse efeito foi diferente tanto para as condições de processo quanto para as direções de caracterização dos filmes. Foi atribuído ao fato de que essa propriedade apresenta uma forte sensibilidade ao grau de orientação e à espessura dos filmes soprados, que são determinados pela taxa de estiramento do filme na direção da máquina. Os filmes processados com baixo Rl apresentam maior resistência ao rasgo e maiores taxas de extensão favorecem a resistência ao rasgo do filme na direção transversal.

As propriedades mecânicas analisadas apresentaram dependências significativas em relação às condições de processo, sendo que os filmes processados a $51 \mathrm{rpm}$ apresentaram melhor desempenho quando comparados àqueles processados a $32 \mathrm{rpm}$, destacando-se as grandes diferenças observadas na resistência ao rasgo. Essas dependências podem ser explicadas pelas diferenças de orientação, espessura e cristalinidade, conforme discutidos ao longo do texto.

\section{Agradecimentos}

Os autores agradecem à FAPESP pelo apoio financeiro (Proc. 00/11193-4 e 00/11194-0) e ao PADCT III/CNPq pela infraestrutura em equipamento. Ao grupo Quimbarra, Mixcim, e Polibrasil pelo fornecimento da carga e aditivos. À $3 \mathrm{M}$ do Brasil por permitir a realização do teste de resistência ao rasgo. Igor Sá Brigagão Perez e Gustavo Berbert Árias pela ajuda na coleta do material reciclado e processamento dos filmes de compósitos.

\section{Referências Bibliográficas}

1. Sousa, J. A. \& Ciminelli, R. R., "Plásticos carregados e reforçados", apostila do curso organizado pela
Associação Brasileira de Polímeros, São Paulo, de 6 a 8 de junho (1995).

2. Rabello, M., "Aditivação de Polímeros”, Artliber Editora Ltda., São Carlos, (2000).

3. Rybnikár. F. - J. Appl. Polym. Sci., 42, p. 2727 (1991).

4. Ren, Z.; Shank, R.A.\& Rook, T.J.- J. Appl. Polym. Sci., 79, p. 1942, (2001).

5. Naiki, M.; et al. - J. Appl. Polym. Sci., 79, p. 1693, (2001).

6. Premphet, K. \& Horanont, P.- J. Appl. Polym. Sci., 76, p. 1929, (2000).

7. García-López, D.; Merino \& Pastor, J. M. - J. Appl. Polym. Sci., 88, p. 947, (2003).

8. Liang, J. - J. Appl. Polym. Sci., 83, p. 1547, (2002).

9. Mai, K; Wang, K; Han, Z. \& Zeng, H. - J. Appl. Polym. Sci., 83, p. 1643, (2002).

10. Mannar, S. M. - Synthetic paper and a process for making it. European Patent 0605938A1, (1994).

11. Desai, S. - Synthetic writable paper- a need of the time", Popular Plastic\&Packaging, Nov., p.45-49, (1994).

12. Ohno, et al. - Synthetic paper with multi-layer structure and excellent printing property. European Patent Appl., No. 95102507.1, december 6, (1995).

13. Nan et al. - Manufacture of biaxially oriented polypropylene synthetic paper with pearl glossiness used as packaging material, involves preheating, annealing and drawing extruded paper in vertical and horizontal directions, International Published under the Patent Cooperation Treaty (PCT), No. WO95/16575, June, 22, (1995).

14. Johnston, G. D. - Laminated printing sheets, International Patent, Derwent, No. TW405002-A, Sept. 11, (2000).

15. Shibata et al. - Processes for treating a surface of a thermoplastic resin film, US Patent\&Trademark Office, No. 6,217,687, April 17, (2001).

16. Lin, F. - Process of 3-layer co-extruded biaxial oriented polypropylene (BOPP) synthetic paper, US Patent, No. $5,552,011$, Sept. 3, (1996).

17. Lin, F. - Process for the production of a biaxially oriented polypropylene synthetic paper of high gloss and easydrying printability, US Patent\&Trademark Office, No. 6,001,290, Dez, 14, (1999).

18. Eckart, et al. - Method of manufacturing filler containing polymer film suitable for printing on, US Patent\&Trademark Office, No. 6.083.443, July, 4, (2000).

19. Lin, F. - The manufacture method of bi-orientation polypropylene (BOPP) synthetic paper fabricated by 
three layer co-extrusion, International Patent, Derwent, No. TW405002-A, Sept. 11, (2000).

20. Huang, H.W. - Synthetic paper and method for its manufacture, European Patent Application No. 95118061.1, May, 14, (1997).

21. Moseley, III. - Polypropylene film suitable for use in inmolde labeling process, US Patent\&Trademark Office, No6,228,316, May 8, (2001).

22. Amon, M. - Opaque polymeric films and processes for making same, US Patent\&Trademark Office, No. 66,183,856, (2001).

23. Ota, S. et al. - Thermal recording media and void-containing polyolefin films for use therein, US Patent\&Trademark Office, No. 6,136,750, Oct. 24, (2000).

24. Cantor, K. - Plástico Moderno., 4883, (2001).

25. Rosato, D. V. \& Rosato, D. V. - Blown molding handbook, Hanser Publishers, p.614-618, (1988).
26. Benning, C. J. - PLASTIC Films for Packaging, Technology, Applications and Process Economics. Technomic Publishing Company, Inc., (1983).

27. Danella, O. - O desenvolvimento e carcaterização de Compósitos de polipropileno para Papel Sintético. Dissertação de Mestrado-DEMa-UFSCar, Setembro, (2001).

28. Legros, N. et al. - "Tensile properties and orientation evolution with processing conditions in polyethylene blown films", in CD do ANTEC, (1998).

29. Patel, R. M. et al. - Polymer Eng. And Science, V.34, N.19, p.1506-1514, (1994).

30. Krishnaswamy, R. K \& Sukhadia, A. M. - Polymer, V.41, p. 9205-9217, (2000).

Enviado: 02/03/04

Reenviado: 20/05/05

Aprovado: 23/06/05 